\title{
THE RESEARCH ON LUCALIBRATION OF GF-4 SATELLITE
}

\author{
WenwenQi, Wei Tan \\ BISME, Beijing institute of space machinery and electronic, 100094, qwwleaves@126.com, blacktanphay@126.com
}

KEY WORDS: GF-4 camera, Lunar radiation calibration

\begin{abstract}
:
Starting from the lunar observation requirements of the GF-4 satellite, the main index such as the resolution, the imaging field, the reflect radiance and the imaging integration time are analyzed combined with the imaging features and parameters of this camera. The analysis results show that the lunar observation of GF-4 satellite has high resolution, wide field which can image the whole moon, the radiance of the pupil which is reflected by the moon is within the dynamic range of the camera, and the lunar image quality can be guaranteed better by setting up a reasonable integration time. At the same time, the radiation transmission model of the lunar radiation calibration is trace and the radiation degree is evaluated.
\end{abstract}

\section{PREFACE}

GF-4 satellite, which is the first high resolution geostationary orbit satellite in the national high resolution earth observation system, is mainly used in disaster reduction, meteorology, forestry and environment and other fields, and it also has great application value in national security, environmental monitoring, weather forecast and so on. GF-4 satellite has the characteristics of relatively fixed observation position, high temporal resolution, wide imaging range and so on, which fills the gap in the field of continuous observation of the earth with high resolution. A perfect complementary earth observation system can be formed by the this satellite with the use of low earth orbit satellites. Meanwhile it can expand the application in the field of astronomy based on its imaging characteristics.

Due to the very stable spectral characteristics of the moon (the surface albedo change rate is about 10-8per year), and the spectrum can cover all the spectral bands of visible light near infrared, making the moon very suitable as a reference source for solar reflection channels. The influence on lunar imaging is not affected by the atmosphere, with the dynamic range of suitable; all of the data can be directly compared with the observation data of different time in a remote sensor and different remote sensors at the same time observation.

\section{LUNAR OBSERVATION INDEX ANALYSIS OF GF-} 4 CAMERA

\begin{tabular}{|c|c|}
\hline Items & Design value \\
\hline $\begin{array}{l}\text { Spectral } \\
\text { range }\end{array}$ & 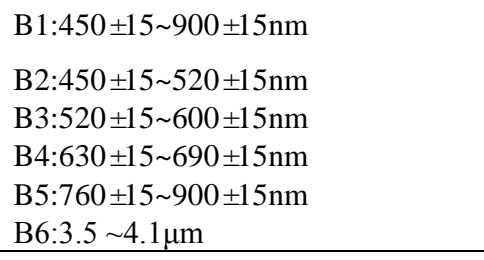 \\
\hline Field & $\begin{array}{l}\text { Visible and near infrared: } 0.8^{\circ} \times 0.8^{\circ} \\
\text { Medium infrared: } 0.66^{\circ} \times 0.66^{\circ}\end{array}$ \\
\hline $\begin{array}{l}\text { Space } \\
\text { resolution }\end{array}$ & $\begin{array}{l}\text { Visible and near infrared:0.28 "/pixel } \\
\text { Medium infrared:2.2"/pixel }\end{array}$ \\
\hline
\end{tabular}

\begin{tabular}{|l|l|}
\hline \multicolumn{1}{|c|}{ Items } & Design value \\
\hline $\begin{array}{l}\text { Dynamic } \\
\text { range }\end{array}$ & $\begin{array}{l}\text { Visible and near infrared:80 degree, } 0.8 \\
\text { Medium infrared:240K } 650 \mathrm{~K}\end{array}$ \\
\hline $\begin{array}{l}\text { Integration } \\
\text { time }\end{array}$ & $\begin{array}{l}\text { Visible and near infrared:0.5ms } \sim 100 \mathrm{~ms} \\
\text { Medium infrared:0.1 } \mathrm{ms} \sim 20 \mathrm{~ms}, \quad \text { the } \\
\text { step is } 0.1 \mathrm{~ms}\end{array}$ \\
\hline
\end{tabular}

Table 1. Camera parameters

\begin{tabular}{|l|c|}
\hline \multicolumn{1}{|c|}{ Items } & value \\
\hline Average orbit radius & $384,403 \mathrm{Km}$ \\
Orbital eccentricity & 0.0564 \\
Perigee distance & $363,300 \mathrm{Km}$ \\
Apogee distance & $405,493 \mathrm{Km}$ \\
Average rotation cycle & 27.32 days \\
Average rotation speed & $1.023 \mathrm{Km} / \mathrm{S}$ \\
Orbital tilt angle & {$\left[28.58^{\circ}, 18.28^{\circ}\right]$} \\
Ascension point & $125.08^{\circ}$ \\
\hline
\end{tabular}

Table 2. Lunar orbit parameters

The indexes that related to the lunar radiance calibration are the lunar resolution, the viewing angle, the moon reflected radiance and the camera output response range. These indexes are estimated with the camera parameters in the following chapters.

\subsection{The lunar resolution}

The lunar resolution can be calculated from the Instantaneous field angle of the camera and the distance between the GF-4 satellite and the moon, which are in a certain interval of [327 thousand, 441 thousand]. This distance is calculated according 
to the lunar orbit and the GF-4 satellite orbit. Here we select a specific value of 384 thousand $\mathrm{km}$ to evaluate the average lunar resolution, the result is showed in the following formula.

Visible : $\operatorname{tand}(0.28 ") * 384 \mathrm{~km}=521 \mathrm{~m}$

Infrared: $\operatorname{tand}(2.2 ") * 384 \mathrm{~km}=4098 \mathrm{~m}$

\subsection{Lunar imaging field}

The diameter of the moon is $3476.4 \mathrm{Km}$, and the maximum number of pixels in one direction of the full moon is as follows.

Visible $: 3476.4 \mathrm{~km} / 521=6672$ pixels

Infrared: $3476.4 \mathrm{~km} / 4098=848$ pixels

The number of pixels in the image field is $10240 * 10240$ of each visible and near infrared spectrum, and $1024 * 1024$ of the infrared spectrum. Therefore, the full disk can be obtained from the image field. In the ideal case that the whole sphere is in the camera's field, the number of pixels of the lunar image in one direction accounts for $65.16 \%$ of the full field, and $82.81 \%$ of the infrared spectrum.

\subsection{The evaluation of the lunar reflect radiance}

The moon, which has the area of about 0.2 square degrees $\left(6.4236 * 10^{-5} \mathrm{sr}\right)$, can be regarded as the Highlighting surface source in the astronomical observation. Usually, lunar observations have no special requirements for the spectral section. The moon reflected radiance depends on the surface reflectivity and reflection angle, and the apparent magnitude of the full moon is up to -12.9 etc. The spectral irradiance of different wavelength in 20 degree phase angle are shown in the following table.

\begin{tabular}{|l|l|}
\hline Wave length(nm) & $\begin{array}{l}\text { Spectral irradiance } \\
(\mu \mathrm{Wm}-2 \mathrm{~nm}-1)\end{array}$ \\
\hline 449.7 & 2.348 \\
499.9 & 2.395 \\
550.0 & 2.633 \\
600.2 & 2.669 \\
650.1 & 2.598 \\
702.8 & 2.474 \\
750.0 & 2.314 \\
850.2 & 1.870 \\
1000.2 & 1.387 \\
\hline
\end{tabular}

Table 3. The spectral irradiance of different wavelength in 20 degree phase angle

According to the spectral irradiance under different lunar phases and the normalized response of the camera, the equivalent radiance of different bands under the different lunar phase can be evaluated. The results are shown in the following table.

\begin{tabular}{|c|c|c|c|c|c|}
\hline \multirow{3}{*}{ Bands } & \multicolumn{5}{|c|}{ The radiance under different angle } \\
\cline { 2 - 6 } & $\begin{array}{c}\text { full } \\
\text { moon }\end{array}$ & $\mathbf{1 0}$ & $\mathbf{3 0}$ & $\mathbf{5 0}$ & $\mathbf{7 0}$ \\
\hline
\end{tabular}

\begin{tabular}{|c|c|c|c|c|c|}
\hline B1 & 18.08 & 17.81 & 15.66 & 12.78 & 9.04 \\
B2 & 2.91 & 2.86 & 2.52 & 2.05 & 1.45 \\
B3 & 3.70 & 3.65 & 3.21 & 2.62 & 1.85 \\
B4 & 2.66 & 2.62 & 2.30 & 1.88 & 1.33 \\
B5 & 4.76 & 4.69 & 4.12 & 3.36 & 2.38 \\
\hline
\end{tabular}

Table 4 . The equivalent radiance of the lunar imaging under different phases $(\mathrm{W} / \mathrm{m} 2 / \mathrm{sr})$

The observational radiance dynamic range of the GF-4 camera is shown in the following table.

\begin{tabular}{|l|l|l|}
\hline \multirow{2}{*}{ Bands } & \multicolumn{2}{|c|}{$\begin{array}{c}\text { Equivalent pupil radiance } \\
\left(\mathbf{W} / \mathbf{m}^{2} / \mathbf{s r}\right)\end{array}$} \\
\cline { 2 - 3 } & $\begin{array}{c}|c| \\
\left(\mathbf{1 0}^{\circ},\right.\end{array}$ & \multicolumn{1}{|c|}{$\left(\mathbf{8 0}^{\circ}, \mathbf{1}\right)$} \\
\hline B1 & 4.95 & 186.44 \\
B2 & 1.46 & 40.42 \\
B3 & 1.1 & 42.3 \\
B4 & 0.5 & 27.1 \\
B5 & 0.5 & 40.9 \\
\hline
\end{tabular}

Table 5 . the radiance dynamic of every bands

According to the comparison of the above two tables, the lunar reflect radiance of the five visible spectral bands and different moon phases are in the range of the camera dynamic.

\subsection{The integration time of the visible and near infrared spectrum}

For the visible and near infrared spectrum, the integration time should be firstly meet the best output in different phase, then the it should match with the integration time of earth observation and the image shift should also be take into consideration.

2.4.1 The optimal integration time under different lunar phases:The output DN of the camera can be estimated according to the equivalent radiance and the laboratory calibration coefficient.

\begin{tabular}{|c|c|c|c|c|c|}
\hline \multirow{2}{*}{$\begin{array}{l}\text { Inte } \\
\text { gration } \\
\text { time }\end{array}$} & \multicolumn{5}{|c|}{ The DN under different angle } \\
\hline & $\begin{array}{c}\text { full } \\
\text { moon }\end{array}$ & 10 & 30 & 50 & 70 \\
\hline $1 \mathrm{~ms}$ & 204.8 & 201.7 & 177.3 & 144.8 & 102.4 \\
\hline $2 \mathrm{~ms}$ & 450.3 & 443.4 & 389.8 & 318.3 & 225.0 \\
\hline $4 \mathrm{~ms}$ & 902.4 & 888.6 & 781.3 & 637.9 & 451.0 \\
\hline $6 \mathrm{~ms}$ & 1363.4 & 1342.5 & 1180.4 & 963.7 & 681.4 \\
\hline $8 \mathrm{~ms}$ & 1832.9 & 1804.9 & 1586.9 & 1295.6 & 916.0 \\
\hline $10 \mathrm{~ms}$ & 2297.1 & 2261.9 & 1988.8 & 1623.7 & 1148.0 \\
\hline $12 \mathrm{~ms}$ & 2786.1 & 2743.5 & 2412.1 & 1969.3 & 1392.4 \\
\hline
\end{tabular}

Table 6. the output DN under different integration time of B1 band 
The International Archives of the Photogrammetry, Remote Sensing and Spatial Information Sciences, Volume XLII-3, 2018 ISPRS TC III Mid-term Symposium “Developments, Technologies and Applications in Remote Sensing”, 7-10 May, Beijing, China

\begin{tabular}{|c|c|c|c|c|c|}
\hline \multirow{2}{*}{$\begin{array}{c}\text { Inte } \\
\text { gration } \\
\text { time }\end{array}$} & $\begin{array}{c}\text { full } \\
\text { moon }\end{array}$ & $\mathbf{1 0}$ & $\mathbf{3 0}$ & $\mathbf{5 0}$ & $\mathbf{7 0}$ \\
\hline $\mathbf{4 m s}$ & 110.8 & 109.1 & 96.0 & 78.3 & 55.4 \\
$\mathbf{1 0 m s}$ & 343.2 & 337.9 & 297.1 & 242.6 & 171.5 \\
$\mathbf{1 6 m s}$ & 583.6 & 574.6 & 505.2 & 412.5 & 291.6 \\
$\mathbf{2 0 m s}$ & 587.8 & 578.8 & 508.9 & 415.5 & 293.8 \\
$\mathbf{3 0 m s}$ & 977.8 & 962.8 & 846.5 & 691.1 & 488.7 \\
$\mathbf{4 0 m s}$ & 1380.5 & 1359.4 & 1195.2 & 975.8 & 689.9 \\
$\mathbf{6 0 m s}$ & 2203.6 & 2169.9 & 1907.8 & 1557.6 & 1101.3 \\
\hline
\end{tabular}

Table 7. the output DN under different integration time of B2 band

\begin{tabular}{|c|c|c|c|c|c|}
\hline \multirow{2}{*}{$\begin{array}{c}\text { Inte } \\
\text { gration } \\
\text { time }\end{array}$} & $\begin{array}{c}\text { full } \\
\text { moon }\end{array}$ & $\mathbf{1 0}$ & $\mathbf{3 0}$ & $\mathbf{5 0}$ & $\mathbf{7 0}$ \\
\hline $\mathbf{4 m s}$ & 204.0 & 200.9 & 176.7 & 144.2 & 102.0 \\
$\mathbf{8 m s}$ & 490.9 & 483.4 & 425.0 & 347.0 & 245.3 \\
$\mathbf{1 0 m s}$ & 640.2 & 630.4 & 554.2 & 452.5 & 319.9 \\
$\mathbf{1 6 m s}$ & 1096.9 & 1080.1 & 949.6 & 775.3 & 548.2 \\
$\mathbf{2 0 m s}$ & 1330.6 & 1310.2 & 1152.0 & 940.5 & 665.0 \\
$\mathbf{3 0 m s}$ & 2101.3 & 2069.2 & 1819.3 & 1485.3 & 1050.2 \\
$\mathbf{4 0 m s}$ & 2835.5 & 2792.1 & 2454.9 & 2004.2 & 1417.1 \\
\hline
\end{tabular}

Table 8. the output DN under different integration time of B3 band

\begin{tabular}{|c|c|c|c|c|c|}
\hline \multirow{2}{*}{$\begin{array}{c}\text { Inte } \\
\text { gration } \\
\text { time }\end{array}$} & \multicolumn{5}{|c|}{ The DN under different angle } \\
\cline { 2 - 6 } & moon & $\mathbf{1 0}$ & $\mathbf{3 0}$ & $\mathbf{5 0}$ & $\mathbf{7 0}$ \\
\hline $\mathbf{4 m s}$ & 169.6 & 167.0 & 146.8 & 119.9 & 84.8 \\
$\mathbf{8 m s}$ & 414.5 & 408.2 & 358.9 & 293.0 & 207.2 \\
$\mathbf{1 0 m s}$ & 543.0 & 534.6 & 470.1 & 383.8 & 271.4 \\
$\mathbf{1 6 m s}$ & 939.6 & 925.2 & 813.4 & 664.1 & 469.6 \\
$\mathbf{2 0 m s}$ & 1032.2 & 1016.4 & 893.7 & 729.6 & 515.9 \\
$\mathbf{3 0 m s}$ & 1680.2 & 1654.4 & 1454.6 & 1187.6 & 839.7 \\
\hline $\mathbf{4 0 m s}$ & 2344.1 & 2308.2 & 2029.4 & 1656.9 & 1171.5 \\
\hline
\end{tabular}

Table 9. the output DN under different integration time of B4 band

\begin{tabular}{|c|c|c|c|c|c|}
\hline \multirow{2}{*}{$\begin{array}{c}\text { Inte } \\
\text { gration } \\
\text { time }\end{array}$} & $\begin{array}{c}\text { full } \\
\text { moon }\end{array}$ & $\mathbf{1 0}$ & $\mathbf{3 0}$ & $\mathbf{5 0}$ & $\mathbf{7 0}$ \\
\hline $\mathbf{4 m s}$ & 132.7 & 130.7 & 114.9 & 93.8 & 66.3 \\
$\mathbf{8 m s}$ & 322.1 & 317.2 & 278.9 & 227.7 & 161.0 \\
$\mathbf{1 6 m s}$ & 728.0 & 716.9 & 630.3 & 514.6 & 363.9 \\
$\mathbf{2 0 m s}$ & 781.2 & 769.2 & 676.3 & 552.2 & 390.4 \\
$\mathbf{3 0 m s}$ & 1279.6 & 1260.0 & 1107.8 & 904.4 & 639.5 \\
$\mathbf{4 0 m s}$ & 1792.3 & 1764.9 & 1551.7 & 1266.9 & 895.8 \\
$\mathbf{5 0 m s}$ & 2309.9 & 2274.6 & 1999.8 & 1632.7 & 1154.4 \\
\hline
\end{tabular}

Table 10. the output DN under different integration time of B5 band

The average reflectance of the lunar surface is $7 \%$, but the highest reflectance of the lunar surface is $24 \%$, the best integration time setting should ensure that the highest reflectance region is not saturation. So the best camera integration time under different phases could be based on this principle. The result is shown in the following tables.

\begin{tabular}{|c|c|c|c|c|c|}
\hline \multirow{2}{*}{ Bands } & \multicolumn{5}{|c|}{ Integration time } \\
\cline { 2 - 6 } & $\begin{array}{c}\text { full } \\
\text { moon }\end{array}$ & $\mathbf{1 0}$ & $\mathbf{3 0}$ & $\mathbf{5 0}$ & $\mathbf{7 0}$ \\
\hline B1 & 4 & 4 & 6 & 6 & 10 \\
B2 & 30 & 30 & 30 & 40 & 60 \\
B3 & 16 & 16 & 20 & 20 & 30 \\
B4 & 20 & 20 & 20 & 30 & 40 \\
B5 & 20 & 20 & 30 & 30 & 40 \\
\hline
\end{tabular}

Table 11. the optimal integration time under different phases

2.4.2 Earth observation integration time:The current GF4 camera in orbit integration time is shown in the following table.

\begin{tabular}{|c|c|c|c|c|c|}
\hline $\begin{array}{c}\text { Bands } \\
\text { Image time }\end{array}$ & B1 & B2 & B3 & B4 & B5 \\
\hline 7:00 9:00 & 12 & 60 & 50 & 50 & 50 \\
9:00 10:30 & 8 & 40 & 30 & 40 & 40 \\
$\begin{array}{c}\text { 10:30 14:0 } \\
0\end{array}$ & 6 & 30 & 20 & 30 & 30 \\
$\begin{array}{c}\text { 14:00 17:0 } \\
0\end{array}$ & 8 & 40 & 30 & 40 & 40 \\
$\begin{array}{c}\text { 17:00 19:0 } \\
0\end{array}$ & 12 & 60 & 50 & 50 & 50 \\
\hline
\end{tabular}

Table 12. On orbit integration time of GF-4 camera(unit:ms) 
2.4.3 The impaction of image shift: The relative speed between the GF- 4 satellite and the moon is 0.219 degrees per minute, and the Instantaneous field angle of the GF-4 camera is 0.28 second. So the integration time should be less than 21.3 $\mathrm{mS}$ in order to control the image shift in one pixel.

From the above analysis, we can see that the reasonable camera output and image quality can be guaranteed by setting the integration time. Because the integration time of multispectral bands is longer than that of image motion, so the actual setting is lower than the theoretical requirement. Nevertheless, the brightness and SNR of the image can be enhanced by the multi frame image enhancement processing.

\section{ANALYSIS OF THE RADIATION TRANSFER MODEL}

To monitor the radiation performance based on the moon, the relative spatial relationship among the sun, the moon and the remote sensors should be established to analyze the solar radiance emitted from the sun to the moon, reflected by moon and received by remote sensor respectively.

\subsection{The irradiance of the sun directly to the moon} In order to calculate the scattering characteristic of the moon, firstly need to calculate the radiance on the surface of the moon produced by the solar radiation. According to Planck blackbody radiation theory, the solar radiation emission $M$ can be expressed as:

$$
\begin{aligned}
M & =\frac{c_{1}}{\lambda^{5}\left[\operatorname{esp}\left(c_{2} / \lambda T\right)-1\right]} \\
E_{S R} & =\frac{r_{S U N}^{2} \cdot M(\lambda)}{d_{M S}^{2}}
\end{aligned}
$$

Where $M$ is the solar radiation emission,

$$
\begin{aligned}
& c_{1}=3.741844 .108 \text { W.m-2. } \mu \mathrm{m} 4 \\
& \left.c_{2}=14388 \mu \mathrm{m} \cdot \mathrm{K}\right)
\end{aligned}
$$

$T$ is the average temperature of the Sun, (also called blackbody temperature, its equivalent temperature $\mathrm{T}$ is 5900),

$r_{\text {SUN }}$ is the radius of the sun, $\left(r_{\text {SUN }}=6.9599 * 10^{8} \mathrm{~m}\right.$

$d_{M S}$ is the average distance between the sun and the

$\operatorname{moon}\left(d_{M S}=1.49597892 \times 10^{11} \mathrm{~m}\right)$.

\subsection{The solar irradiance reflected by the moon}

$$
E_{\text {lua }}=E_{S R} \sqcap \rho(\alpha, \lambda)
$$

Although the moon is not uniform, the average reflectance of the lunar surface is quite stable in the same phase. For the same spectrum, the average reflectivity can be viewed as a function with the moon and the change of wavelength is assumed to be $p\left(\alpha_{x}^{\lambda}\right)$.

\subsection{The lunar irradiance received by the camera}

The irradiance of the lunar space optical camera within the range of the spectrum of the sun can be expressed as the following formula.

$$
E_{R C}=\frac{E_{l u a} \cdot r_{l u a}^{2} \cdot \cos \theta_{R C}}{d_{R C}^{2}}
$$

Where $r_{\text {lua }}$ is the lunar radius.

$\theta_{R C}$ is the lunar phase (it equals 0 in the full moon)

$d_{R C}$ is the distance between the moon and the satellite.

\subsection{The equivalent radiance under different bands}

$$
L_{C-B i}=\int_{\lambda_{1}}^{\lambda_{2}} R_{C-B i}(\lambda) \cdot E_{R C}(\lambda) d \lambda / \int_{\lambda_{1}}^{\lambda_{2}} R_{C-B i}(\lambda) d \lambda
$$

Where $R_{C-B i}(\lambda)$ is the normalized spectral response function of NO.i spectral.

\section{THE TRACEABILITY ANALYSIS OF THE LUNAR RADIATION CALIBRATION OF GF-4 SATELLITE}

In the radiometric calibration transfer link of the GF-4 satellite, the radiance received by remote sensing can be traced back to SI. Compared with the traditional radiation transfer method traced to the ground, the lunar radiative transfer reduces the transfer steps and reduces the uncertainty in the baseline transfer process.

The error sources of lunar radiation transmission are mainly divided into five parts: distance error, angle error, measurement uncertainty of solar radiation, error of lunar radiative transfer model and error of stray light effect. The analysis of the error of radiative transfer is shown in the following diagram.

\subsection{The uncertainty of the solar irradiance}

The uncertainty of the solar irradiance is $0.25 \%$.

\subsection{The Measurement uncertainty of solar irradiance} reach to the surface of the moon

The solar irradiance that reaches the surface of the moon varies with the distance between the moon and the sun. Because the variation of the distance could be neglected relative to the distance itself, so the uncertainty of the term could be neglected.

\subsection{Distance measurement uncertainty between the entrance pupil of the remote sensor and the moon}

It is assumed that the distance calculation error is $1 \mathrm{~km}$, and the uncertainty of the distance is different according to the difference of the distance measured. When the nearest distance between the moon and the satellite is $3.57 * 105 \mathrm{~km}$, the uncertainty is negligible. 


\subsection{Phase angle error}

Assuming the angle measurement error of 0.2 degree, the uncertainty of the lunar phase can be preliminary calculated is less than $0.6 \%$.

\subsection{Uncertainty of the average lunar reflectance model}

The error of the average lunar reflectivity is related to the testing method. At the present stage, the relative value of the uncertainty can reach $1 \%$ and the absolute value is $5 \%$ by referring to the global lunar radiation model. With the accumulation of GF-4 camera calibration data, a higher surface emissivity model is expected to be established.

The error is mainly affected by the satellite attitude pointing accuracy, which is better than 0.01 degrees. Assuming that the distance between the moon and the satellite is $3.57 * 10^{5} \mathrm{~km}$, the surface position of the pointing error, which could be derived according to the pointing accuracy, is about $61 \mathrm{~km}$. According to the analysis of the imaging field, only the moon position could be affected by the pointing error. On the basis of relative radiation correction, the uncertainty of the term can be ignored.

\subsection{Uncertainty of the dispersion spot formatted on the lunar surface}

The uncertainty of the spot will affect the output response of the local area, but the error can be neglected based on the full disk integration.

\subsection{Non-uniformity of the detector}

The non-uniformity of detectors is mainly determined by the relative radiometric correction accuracy. The accuracy of relative radiation correction of GF-4 cameras can reach $1.5 \%$ (output response is higher than 20\%).

\begin{tabular}{|l|c|c|}
\hline \multicolumn{1}{|c|}{ Error resource } & $\begin{array}{c}\text { Accuracy of } \\
\text { response } \\
\text { stability } \\
\text { monitoring }\end{array}$ & $\begin{array}{c}\text { Accuracy of } \\
\text { radiation } \\
\text { calibration }\end{array}$ \\
\hline $\begin{array}{l}\text { Uncertainty of measurement } \\
\text { of solar radiation }\end{array}$ & $0.25 \%$ & $0.25 \%$ \\
$\begin{array}{l}\text { Lunar phase error } \\
\text { The uncertainty of the lunar } \\
\text { reflect radiance }\end{array}$ & $0.6 \%$ & $0.6 \%$ \\
Non-uniformity of detector \\
Total error
\end{tabular}

Table 14. Accuracy analysis of radiometric calibration of visible spectrum

\section{CONCLUSION}

Starting with the requirement of GF-4 satellite to lunar observation, the imaging characteristics of the lunar observation are analyzed in combination with the imaging features and imaging parameters of the camera. The analysis results show that the lunar observation of GF-4 satellite has high resolution, wide field which can image the whole moon, the radiance of the pupil which is reflected by the moon is within the dynamic range of the camera, and the lunar image quality can be guaranteed better by setting up a reasonable integration time. At the same time, the radiation transmission model of the lunar radiation calibration is traced and the radiation degree is evaluated.

\section{REFERENCES}

Huang Wenxin, Zhang Liming, Si Xiaolong, et al, 2016.Theory and precision maintenance method of on-orbit reflectance calibration [J]. In: Acta Optica Sinica, 36(6):0623001.

Qiu Ganggang, Li Xin, Wei Wei, et al, 2016. Experiment and analysis of on-orbit radiometric calibration for remote sensors based on in-site automated observation technology [J]. In:Acta Optica Sinica, 36(7):0701001.

Delwart S, Bourg L, 2009. Radiometric calibration of MERIS[C]. In:Proceeding of SPIE Conference on Sensors, Systems, and next-Generation Satellites XV, Paris: University of Paris, 7474:1-12.

Slater P N, Biggar S F, Thome K J, 2009. Vicarious Radiometric Calibrations of EOS Sensors[J]. In:Journal of Atmospheric \& Oceanic Technology, 13(2).

Zheng Xiaobing, 2011. High-accuracy radiometric calibration of satellite optical remote sensor [J]. In:Spacecraft Recovery \& Remote Sensing, 32(5), pp.36-43.

Zhang Wanying, Wang Tao, Chen Fansheng, 2017. Research on determining methods of observed stellar in on-board radiometric calibration [J]. In:Chinese Space Science and Technology, 37(3),pp.71-76. 